\title{
Medical image computing and image-based simulation: recent developments and advances in Germany
}

\author{
Heinz Handels - Hans-Peter Meinzer • \\ Thomas M. Deserno • Thomas Tolxdorff
}

Published online: 17 May 2014

(c) CARS 2014

\section{Introduction}

Medical image computing has become a key technology in image-based medical diagnostics and image-guided therapy. In medical diagnostics, methods of image computing enable new insights into the patient's anatomy and physiology and support the extraction of semantic objects (organs, vessels, tumors, etc.) as well as quantitative parameters (tumor volume, etc.) from the image data. In the field of softwareassisted and navigated therapy, medical image computing has opened up new perspectives for patient treatment. Although methods and systems of medical image computing are yet applied in practice, their grade of automation, accuracy, reproducibility, and robustness has to be increased to meet the requirements in clinical routine [1-7].

H. Handels $(\bowtie)$

Institute of Medical Informatics, University of Lübeck, Ratzeburger Allee 160, 23562 Lübeck, Germany

e-mail: handels@imi.uni-luebeck.de

H.-P. Meinzer

Department of Medical and Biological Informatics,

German Cancer Research Center (DKFZ),

Im Neuenheimer Feld 280, 69120 Heidelberg, Germany

e-mail: h.p.meinzer@dkfz-heidelberg.de

T. M. Deserno

Department of Medical Informatics, Uniklinik RWTH Aachen,

52057 Aachen, Germany

e-mail: deserno@ieee.org

T. Tolxdorff

Institute of Medical Informatics, Charité - Universitätsmedizin Berlin, Hindenburgdamm 30, 12200 Berlin, Germany

e-mail: thomas.tolxdorff@charite.de

\section{Methods}

For this special issue, selected authors of the German Conference on Medical Image Computing 2013 (Bildverarbeitung für die Medizin, BVM 2013, http://bvm-workshop.org) were invited to submit a manuscript on their latest developments and results for possible publication in this special issue. The BVM 2013 was hosted by the Department of Medical and Biological Informatics, German Cancer Research Center (DKFZ), Heidelberg, with 200 participants from engineering and computer science, medicine, and industry. The invitation was based on the blinded peer review process of the conference and the outstanding presentation. After international reviewing of the journal submissions, eight excellent papers were assembled to describe recent advances in the field of medical image computing and image-based simulation.

\section{Results}

Recent developments in medical image reconstruction, registration, analysis, and image classification as well as imagebased model generation and model-based simulation are presented in this special issue, emphasizing the variety of methods used in this challenging field and showing contributing results in several medical applications.

Xia et al. [8], present a calibration method for the approximated truncation robust algorithm for computed tomography (ATRACT), a reconstruction technique for region of interest imaging in C-arm computed tomography. The interesting method enables to reconstruct images without the use of any explicit extrapolation or prior knowledge, even in presence of severe data truncation. In the paper, Xia et al. present a scaling calibration method that reduces scaling artifacts in ATRACT algorithm, significantly. 
The next three contributions [9-11] address different aspects of medical image analysis and image-based simulation in the field of computer-assisted diagnostics. In [9], medical image analysis networks are used to quantify changes in language-related brain areas in autism spectrum disorders. Goch et al. mainly focus on assessing the concept of network centrality as a tool to perform brain structure specific analysis. Krüger et al. [10] present a method to support multimodal breast diagnostics analyzing 2D mammography and 3D MRI of the female breast. An iterative closest point (ICP)based B-spline approach is proposed to compensate large differences in breast deformation between mammography and MRI. The novel deformation model is applied to simulate mammographic breast compression in 3D MRI, an essential step toward the automatic detection of corresponding structures in 2D mammography and 3D MRI of the breast. In [11], medical image analysis and pattern recognition methods are combined for cell versus background classification on multiple cell lines in bright-field microscopy. Using random forest and support vector machine classifiers, Mualla et al. show that replacing the defocused image with the local phase of the monogenic signal improves the cell-background classification rate.

The contributions [12-15] of this special issue illustrate the impact of medical image computing and image-based simulation techniques in the field of computer-assisted therapy. Berkels et al. [12], present an approach to co-register intraoperative microscopy with preoperative MRI to support intraoperative neuro-navigation. With this method, they address a key problem in neuro-navigation, the change in configuration of the brain, and the brain shift resulting from opening the dura mater. The variational registration algorithms enable robust classification of sulci on cortical surfaces extracted from MR images and guide user marking of most prominent sulci on the microscope image. Marx et al. [13], present a novel 4D MRI-based lung motion model to support $4 \mathrm{D}$ radiation therapy of lung tumor patients. The proposed methods enable the use of 4D MRI instead of 4D $\mathrm{CT}$ to acquire respiratory motion information for the radiotherapy planning process, significantly reducing the dose exposure of the patient. From 4D MRI, a time-continuous $\mathrm{B}$-spline-based model of the average patient-specific respiratory motion is derived and applied to simulate 4D CT data based on a static 3D CT using nonlinear registration methods. März et al. [14] present an extension of the Medical Imaging Interaction Toolkit (MITK) for computer-assisted ultrasound (US) interventions. Using the open-source software module MITK-US, a new concept for US-guided needle insertion was implemented successfully. It is shown that MITK-US provides a useful rapid prototyping environment for USbased computer-aided interventions. Mastmeyer et al. [15] present a virtual reality simulator for the visio-haptic training of percutaneous transhepatic catheter drainage punctures.
The paper is focused on a software framework enabling systematic evaluation of the haptic forces applied to the virtual needle during simulated punctures. Ray casting techniques are used to select optimal needle paths from the skin to the target structures. Along these selected paths, force output comparisons between an established reference system and new haptic force algorithms using haptic volume rendering in un-segmented virtual bodies are carried out.

\section{Discussion}

The selected papers give an impression of the breadth and heterogeneity of new developments in the field of medical image computing and image-based simulation, as presented at the German Conference on Medical Image Computing (BVM 2013). New methods for image reconstruction, quantitative image analysis, multimodal correspondence analysis, as well as new approaches for model-based 4D radiation therapy are assembled. Furthermore, novel methods, software toolkits, and image-based virtual reality simulations are described to support patient-specific preparation, training, and execution of computer-assisted interventions.

\section{References}

1. Deserno TM, Handels H, Meinzer H-P, Tolxdorff T (2013) Recent advances in 3D medical image generation and analysis. Curr Med Imag Rev 9(2):77-78

2. Handels H, Deserno TM, Meinzer H-P, Tolxdorff T (2012) Image analysis and modeling in medical image computing-recent developments and advances. Methods Inf Med 5:395-397

3. Deserno TM, Aach T, Amunts K, Hillen W, Kuhlen T, Scholl I (eds) (2011) Advances in medical image processing. Comput Sci Res Dev 26(1):1-3

4. Handels H, Ehrhardt J (2009) Medical image computing for computer supported diagnostics and therapy-advances and perspectives. Methods Inf Med 48:11-17

5. Tolxdorff T, Deserno TM, Handels H, Meinzer HP (2009) Advances in medical image computing. Methods Inf Med 48(4):311-313

6. Handels H, Horsch A, Meinzer HP (2007) Advances in medical image computing. Methods Inf Med 46(3):251-253

7. Horsch A, Deserno TM, Handels H, Meinzer HP, Tolxdorff T (2008) Editorial, IJCARS special issue: BVM 2007 German conference on medical image processing. Int J CARS 2(5):253-254

8. Xia Y, Dennerlein F, Bauer S, Hofmann H, Hornegger J, Maier A (2014) Scaling calibration in region of interest reconstruction with the 1D and 2D ATRACT algorithm. Int J CARS. doi:10.1007/ s11548-014-0978-z

9. Goch CJ, Stieltjes B, Henze R, Hering J, Poustka L, Meinzer HP, Maier-Hein KH (2014) Quantification of changes in language related brain areas in autism spectrum disorders using large-scale network analysis. Int J CARS. doi:10.1007/s11548-014-0977-0

10. Krüger J, Ehrhardt J, Bischof A, Handels H (2014) Simulation of mammographic breast compression in 3D MR images using ICP-based B-spline deformation for multimodality breast cancer diagnosis. Int J CARS. doi:10.1007/s11548-014-0976-1 
11. Mualla F, Schöll S, Sommerfeldt B, Hornegger J (2014) Using the monogenic signal for cell-background classification on multiple cell lines in bright-field microscope images. Int J CARS. doi:10. 1007/s11548-013-0969-5

12. Berkels B, Cabrilo I, Haller S, Rumpf M, Schaller C (2014) Coregistration of intra-operative photographs and pre-operative MR images. Int J CARS. doi:10.1007/s11548-014-0979-y

13. Marx M, Ehrhardt J, Werner R, Schlemmer H-P, Handels H (2014) Simulation of spatiotemporal CT data sets using a 4D MRI-based lung motion model. Int J CARS. doi:10.1007/s11548-013-0963-y
14. März K, Franz AM, Stieltjes B, Seitel A, Radeleff B, Meinzer HP, Maier-Hein L (2014) Computer-assisted ultrasound interventions with the medical imaging interaction toolkit. Int J Comput Assist Radiol Surg

15. Mastmeyer A, Hecht T, Fortmeier D, Handels H (2014) Ray-casting based evaluation framework for haptic force feedback during percutaneous transhepatic catheter drainage punctures. Int J CARS. doi:10.1007/s11548-013-0959-7 